\title{
Adaptive Refinements in BEM
}

S. Gómez Lera, M. Cerrolaza and E. Alarcón

E. T.S. Industrial Engineers, Polytechnical University of Madrid, Madrid 28006, Spain

\section{INTRODUCTION}

Accuracy estimates and adaptive refinements is nowadays one of the main research topics in finite element computations $[6,7,8$, $9,11]$. Its extension to Boundary Elements has been tried as a means to better understand its capabilities as well as to impro ve its efficiency and its obvious advantages. The possibility of implementing adaptive techniques was shown $[1,2]$ for $h$-conver gence and $\mathrm{p}$-convergence respectively. Some posterior works $[3,4$ $5,10]$ have shown the promising results that can be expected from those techniques. The main difficulty is associated to the reasonable establishment of "estimation" and "indication" factors related to the global and local errors in each refinement. Although some global measures have been used it is clear that the reduction in dimension intrinsic to boundary elements $(3 \mathrm{D} \rightarrow 2 \mathrm{D}$ : $2 \mathrm{D} \rightarrow 1 \mathrm{D})$ could allow a direct comparison among residuals using the graphic possibilities of modern computers and allowing a point-to-point comparison in place of the classical global approaches. Nevertheless an indicator generalizing the well known Peano's one has been produced.

This work deals with the application of BEM p-convergence approach to the analysis of potential and elasticity problems, showing that the establishment of a suitable convergence criteria is the cornerstone question in such approach. Moreover, it is still an open question and, in the author's opinion, deserves a great amount of research. Unfortunately, the mathematical support for the convergence in the BEM p-adaptive approach is still lacking, which enhances the unavoidable requirement of research in that way. 
In the BEM p-adaptive approach, the geometry and boundary condition representation are independent of the process of analysis, allowing the use of geometry preprocessors in order to minimise error sources derived from non-regular discretisations.

The present development uses "serendipity" functions defined over linear (2-D) and surface (3-D) variable-number-nodes boundary elements, to adjust both geometry and Boundary conditions in an independent way (Fig. 1).

"Self-solvables" corners and edges, produced by special boundary conditions, are identified and treated separately from the global process, reducing the number of operations required to reach the final solution.

Variable-number-nodes elements has the advantage of great flexibility in geometry and boundary conditions definition. Moreover, mid side nodes (2-D,3-D) and internal nodes (3-D) do not affect the interpolation approach selected for adjust the problem unknowns.

\section{INTERPOLATION FUNCTIONS: HIERARCHICAL APPROACH}

The first step of the method is the choice of the interpolation family functions. Our criterion is to select the greatest boundary elements compatible with both geometry and boundary condition discontinuities. This choice minimizes the number of boundary elements and, of course, the operations required in the analysis procedure.

Next, the hierarchy of the interpolation functions to be used in the successive approximations process is defined.

In previous papers treating with plane potential problems the Peano's family was used. In those cases it was observed that for high order polynomial degree the convergence rate was slower than for low degrees. Although that problem deserves more research, in this paper we have decided to use the Legendre family of interpolation functions, defined by:

$$
\begin{gathered}
N_{\circ}(\xi)=\frac{1}{2}(1+\xi) ; \quad N_{1}(\xi)=\frac{1}{2}(1-\xi) \\
N_{p}(\xi)=\frac{1}{(p-1) !} \frac{1}{2^{p-2}} \frac{d^{p-2}}{d \xi^{p-2}}\left[\left(1-\xi^{2}\right)^{p-1}\right] ; p \geqq 2
\end{gathered}
$$

with appropiate combinations based on the well known tensorial products for 3-D analysis. 
These functions are defined over unidimensional elements for 2-D and over either edges or surface elements for 3-D, requiring a careful strategy when programming the adaptive process in order to consider the respective refinements in a sequential way.

For 2-D analysis (i.e. unidimensional elements) the new interpolations functions are "bubbles" inside the element. The problem arises when increasing the interpolation order and, in consecuence, the collocation points move toward the corners. In these cases, numerical integrations of singular kernels expe riment an appreciable decrease of accuracy. The same occurs in 3-D domains, when collocation points move near either an edge or a surface element.

\section{COLLOCATION POINTS}

As it is well known, BEM integral equations are generated by collocating the fundamental solution (Green's function for the whole space) in selected boundary points. In the particular case of isoparametric BEM, the same set of points to define geometry, boundary conditions, interpolation points and collocation points is used thus producing undesiderable effects when the method must fit into special local situations.

However, in the BEM p-adaptable version we have complete freedom for selecting nodal points. The main idea is to choose the collocation points where the interpolation functions give their maximum value, in order to reinforce the corresponding dominant element within the influence matrix. Also, collocation points must be placed as far apart as possible to avoid ill-con ditioning in the system of equations. Thus, for 2-D analysis, linear functions are defined in corner nodes and "bubble" functions are placed in collocation points, as showing in figure 1.

In 3-D analysis, bilinear functions are defined in corner nodes and dd/even functions are placed on points belonging to edges/elements respectively.

Searching for the best place to define collocation points and its importance in BEM p-adaptive approach is an open question. 

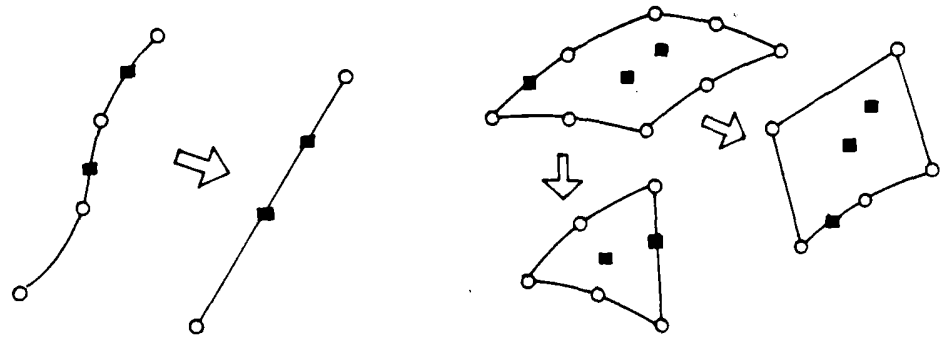

Fig. 1. Variable-number-nodes elements for 2-D and 3-D domains 0 : "geometric" nodes : collocation points.

INFLUENCE MATRICES AND ASSEMBLAGE OF COEFFICIENTS IN ADAPTIVE ANALYSIS

As it is well known, the influence matrices produce in adaptive analysis are nested, i.e., all the previous integrals remain valid for the current refinement step and only some new integral coefficients from new interpolation functions must be calculated and assembled. Thus, when one proceeds to integrate ele ment-by-element, it is only necessary to generate the integrals corresponding to: a) new interpolation functions when they are viewed from collocation points existing in all previous steps (including corners) and b) all interpolation functions, including linear (2-D) and bilinear (3-D), when they are viewed from new collocation points in the actual refinement stage.

This procedure, together with the fact that the process is made element-by-element, reduces to a minimum the computational effort.

In order to guide the assemblage process, the computer generates a set of alfanumeric codes for the corner nodes (in 2-D and 3-D domains) from previous information given for the bounda $r y$ elements. For instance, in two dimensional elasticity, a cor ner node between one element with all displacements prescribed (UV) and another one with the tractions prescribed (ST) would be a "UVST" corner and so on.

The new interpolation functions added for refinement will automatically introduced in to the influence matrix by knowing the boundary element identification and the corners code described above. This process is simpler for potential problems than for elasticity due to the fact that in 2-D elasticity, each new collocation point reflects the refinement of only one unknown and, of course, if could be necessary to refine both of then over an element. In such cases, two new collocation points must be defined for account two new interpolation functions. 
In what follows, we will describe briefly the parameters which control the adaptive refinement process, the so-called "indicators" and "estimators". In order to be clear, only potential problems will be considered herein.

Their extension to elasticity do not present special complications. The reader interested in a more detailed formulae is refered to $[5,6]$.

The representation formula for potential problems could be written as follows:

$$
\begin{gathered}
C \phi(P)+\int_{S_{2}} \phi(Q) \cdot q^{*}(P, Q)-\int_{S_{1}} q(Q) \cdot \phi^{*}(P, Q)= \\
=-\int_{S_{1}} \phi^{\circ}(Q) \cdot q^{*}(P, Q)+\int_{S_{2}} q^{\circ}(Q) \cdot \phi^{*}(P, Q)
\end{gathered}
$$

where $\phi(Q)$ and $q(Q)$ are potential and flux unknown fields at $Q$ and the boundary $S$ is $\mathrm{S}_{1} \mathrm{U} \mathrm{S}_{2}$.

Equation (2) is subjected to mixed boundary conditions:

$$
\phi=\phi^{\circ} \text { on } \mathrm{s}_{1} ; \quad \mathrm{q}=\mathrm{q}^{\circ} \text { on } \mathrm{s}_{2}
$$

The function $\phi^{*}$ is the fundamental solution for the Laplace equation, collocated at $\mathrm{P}$ :

$$
\phi_{(P, Q)}^{*}=-\frac{1}{4 \pi r(P, Q)} ; \quad q_{(P, Q)}^{*}=\frac{\partial}{\partial n_{Q}}\left[\phi^{*}(P, Q)\right]
$$

and $\mathrm{P}$ and $\mathrm{Q}$ are boundary points. $\mathrm{C}$ is a constant depending on boundary geometry at $P$.

$$
\begin{aligned}
& \text { Collecting terms in (2): } \\
& \qquad L_{1} q(P)+L_{2} \phi(P)+p(P)=0
\end{aligned}
$$

where we state the following DEFINITION:

$$
\begin{aligned}
& L_{1} f(P)=-\int_{S_{1}} f(Q) \phi^{*}(P, Q) \\
& L_{2} f(P)=C f(P)+\int_{S_{2}} f(Q) q^{*}(P, Q) \\
& P(P)=\int_{S_{1}} f^{O}(Q) q^{*}(P, Q)-\left.\int_{S_{2}} \frac{\partial f}{\partial n}\right|_{Q} \phi^{*}(P, Q)
\end{aligned}
$$


Unknown potential and flux are approximated over $\mathrm{S}_{2}$ and $S_{1}$ respectively through:

$$
\begin{aligned}
& \phi \sim \bar{\phi}=a_{1} N_{1}+a_{2} N_{2}+\ldots+a_{n} N_{n} \text { on } s_{2} \\
& q \sim \bar{q}=b_{1} N_{1}+b_{2} N_{2}+\ldots+b_{n} N_{n} \text { on } s_{1}
\end{aligned}
$$

where $a_{j}$ and $b_{j}$ are parameters to be determined, where as $\mathrm{N}_{j}$ are elements of hierarchical family functions.

From these approximations, two errors are derived:

$$
\begin{aligned}
& e_{1}=q-\bar{q} \text { on } s_{1}|| e_{1}=0 \text { on } s_{2} \\
& e_{2}=\phi-\hat{\phi} \text { on } s_{2}|| e_{2}=0 \text { on } s_{1}
\end{aligned}
$$

The "residual" $r$ is obtained substituting (7) in to (2):

$$
r=L_{1} \hat{q}+L_{2} \hat{\phi}+p
$$

It must be emphasized that residual $x$ is zero at collocation points and, with (9), $r$ can be calculated at any other boundary point.

From (8) we can write:

$$
\begin{aligned}
& L_{1} \bar{q}=L_{1} q-L_{1} e_{1} \\
& L_{2} \hat{\phi}=L_{2} \phi-L_{2} e_{2}
\end{aligned}
$$

Finally, afther some algebra, we obtain:

$$
\begin{aligned}
& r=-L_{1} e_{1}=r_{1} \text { on } s_{1} \\
& r=-L_{2} e_{2}=r_{2} \text { on } s_{2}
\end{aligned}
$$

Although the BEM approach is a collocation method, a global "energy" error can be established through techniques resembling Galerkin method:

$$
\begin{aligned}
\|e\|_{E}^{2} & =\int_{S_{1}} e_{1}\left(L_{1} e_{1}\right)+\int_{S_{2}} e_{2}\left(L_{2} e_{2}\right)= \\
& =-\int_{S_{1}} e_{1} r_{1}-\int_{S_{2}} e_{2} r_{2}
\end{aligned}
$$

or, developing a bit further:

$$
\begin{aligned}
& \|\mathrm{e}\|_{E}^{2}=\int_{S_{1}} e_{1}(P)\left[-\int_{S_{1}} e_{1}(Q) \phi^{*}(P, Q)\right]+C \int_{S_{2}}\left[e_{2}(P)\right]^{2}+ \\
& \quad+\int_{S_{2}} e_{2}(P)\left[\int_{S_{2}} e_{2}(Q) \cdot q^{*}(P, Q)\right]
\end{aligned}
$$


An interesting property of (13) is obtained considering that flux is known and potential is refined (the same applies when potential is known and flux is refined) by adding a new interpolation function:

$$
\phi \sim \hat{\hat{\phi}}=\hat{\phi}+a_{n+1} N_{n+1}
$$

or, substituting into (8):

$$
e_{2}=\phi-\hat{\phi} \sim a_{n+1} N_{n+1}
$$

Now, using (13), we obtain:

$$
\|\left. e\right|_{E} ^{2}=-a_{n+1} \int_{S_{2}} N_{n+1} r_{2}
$$

It can be seen that the energy error is related to the dot product of $x$ and $\mathrm{N}_{n+1}$. In fact, this error works to indicate the usefulness of introducing the new function but not to estimate the actual value of the residual. Now, it is possible to establish a relationship between error and residual.

Manipulating conveniently the preceding formulas we arrive at the desired expression for the indicator:

$$
\|e\|_{E}^{2}=\frac{\left[\int N_{n+1} r_{2}\right]^{2}}{\int N_{n+1} L_{2} N_{n+1}} \text { on } s_{2}
$$

with a similar expression for boundary $\mathrm{S}_{1}$.

The estimators used in this work are not completely established, but as can be seen from numerical examples, they work good enough. The first of them is a "residual estimator" and takes the form:

$$
\|E\|^{2}=\frac{1}{C} \sum_{i} \int_{S_{j}} r_{i}^{2}(P) d_{i}
$$

The other one is the summation of fluxes over the whole boundary, which must be zero:

$$
|| E||^{2}=\Sigma\left[\int_{s_{i}} q_{i}\right]^{2}
$$

NUMERICAL RESULTS. CONVERGENCE STUDY

In order to evaluate the convergence and the versatility of the numerical procedure developed, the BEM p-adaptive approach presented in the previous sections is now applied to three illus- 
trative examples. This examples were analysed with program "PTADAP", written in Fortran 77 and running on a UNIVAC machine, specially developed for this research.

\section{a) Regular cube}

The figure 2 shows the theoretical solution and the results of successive refinements over the "developed" cube.

Figures 3 illustrate the drastic reduction of the residuals on the elements when new interpolation functions are collocated over the edges, as expected.

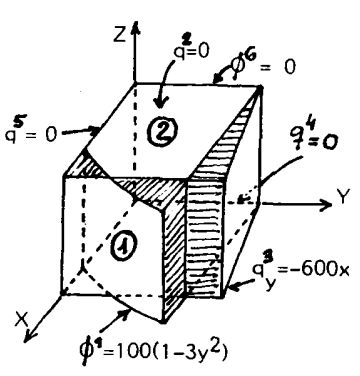

(a)

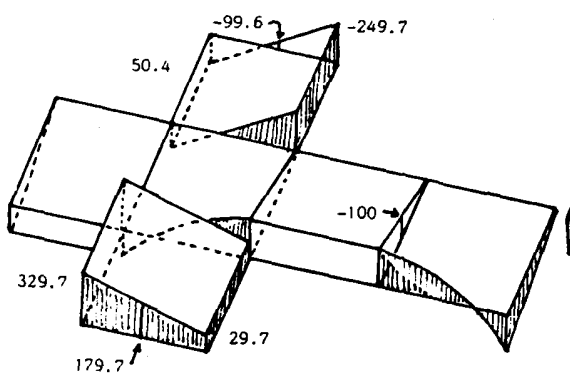

(c)

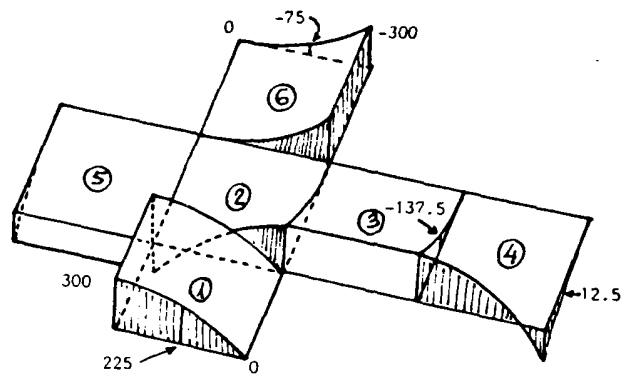

(b)

Fig. 2. Regular cube example:
(a) boundary conditions
(B) theoretical solution: $\phi=100\left(x^{3}-3 x y^{2}\right)$
(c) bilinear interpolation solution
(d) quadratic interpolation solution 


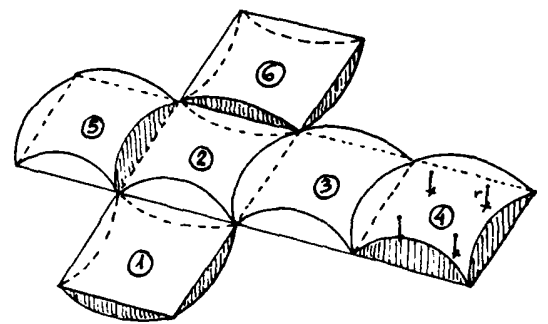

\begin{tabular}{|c|c|c|c|c|c|c|}
\hline Elem & 1 & 2 & 3 & 4 & 5 & 6 \\
\hline \multirow{4}{*}{$r$} & -10.42 & 10.07 & 10.81 & 20.30 & 10.73 & -13.65 \\
& -10.42 & 20.30 & 20.04 & 10.07 & 10.73 & -13.82 \\
& -10.36 & 10.03 & 10.81 & 20.55 & 19.84 & -13.65 \\
& -10.30 & 20.55 & 20.04 & 10.37 & 19.84 & -13.82 \\
\hline
\end{tabular}

(a)

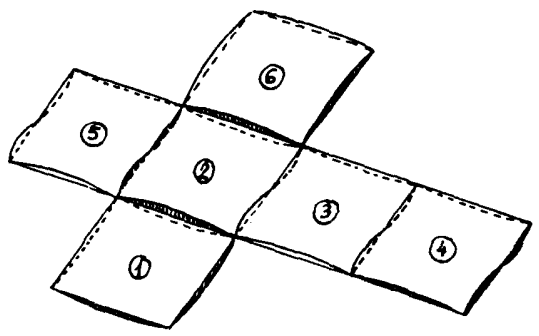

\begin{tabular}{|c|c|c|c|c|c|c|}
\hline Elem & 1 & 2 & 3 & 4 & 5 & 6 \\
\hline \multirow{3}{*}{$r$} & -1.57 & -4.02 & -3.92 & 3.89 & -4.01 & 1.38 \\
& -1.57 & 3.89 & 4.27 & -4.02 & -4.01 & 1.21 \\
& -1.53 & -4.06 & -3.92 & 4.14 & 4.07 & 1.38 \\
& -1.53 & 4.14 & 4.27 & -4.06 & 4.01 & 1.21 \\
\hline
\end{tabular}

(b)

Fig. 3. Evolution of residuals in cube example:

(a) bilinear interpolation

(b) quadratic interpolation

b) Hollow cylinder

This example was analysed using both BEM p-refinement and h-refinement approaches. Figure 4 shows boundary conditions and theoretical solution. Figure $5 \mathrm{a}$ shows the logarithm evolution for the quadratic error proposed in expression (18), which is used as a global estimator for the solution convergence, ver sus the logarithm of $1 / \mathrm{NDF}$ (NDF being the total number of integral equations at each refinement stage). Observe that p-refine ment convergence (adaptive approach) is about 1.5 times faster than selective $h$-refinement convergence. This is a relevant and promising fact for future research.

Figure $5 \mathrm{~b}$ shows the evclution of the summation of fluxes over the whole boundary (again in logarithm scale and squared to avoid the effect of sign), versus the logarithm of 1 /NDF. P-refinement convergence (adaptive approach) is again faster. Observe that p-refinement convergence (complete approach) also ensures the convergence but, in this case, it's about 1.25 times slower than the selective h-refinement approach and 1.92 times slower than p-refinement convergence (adaptive approach). However, this kind of estimator must be used carefully because it could give wrong results when using over more complicated geometries. 


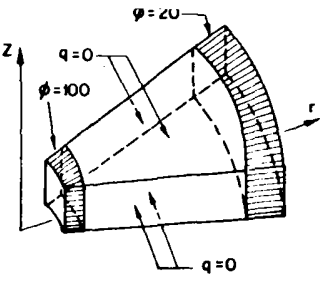

(a)

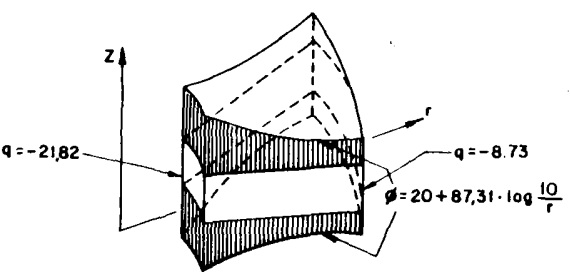

(b)

Fig. 4. Hollow cylinder example:

(a) boundary conditions

(b) theoretical solution

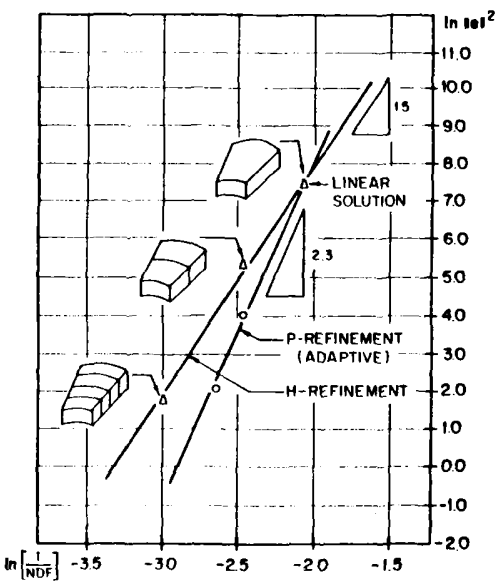

(a)

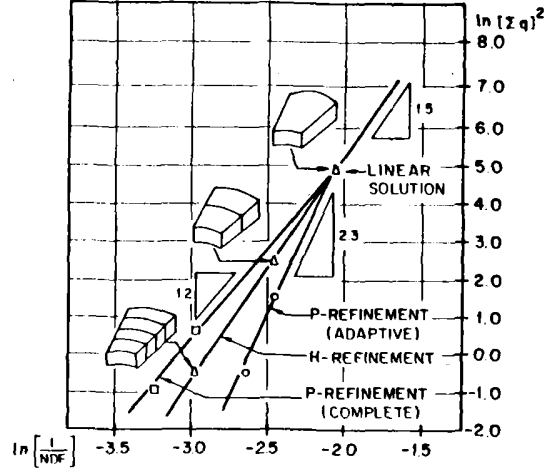

(b)

Fig. 5. Convergence in Hollow cylinder:

(a) Residual error

(b) summation of fluxes

c) Simplified pressure vessel

A simplified wedge sector of an axially symmetric pressure vessel is now analysed to determine the temperature and flux fields over the boundary. The body geometry is "extracted" from a parallelepiped body on which both exact solution and boundary conditions are known.

In this example both $\mathrm{p}$ and $\mathrm{h}$-convergence approaches were used. As expected, p-convergence is again better than $h$-conver gence, showing faster rates of convergence (see Fig. 7). 
This problem was analysed using bilinear interpolation functions over single-curved and plane surface quadrilateral variable-number-nodes elements, defining the meshes shown in Figure 6. Mesh $A$ was based on 82 nodes and 54 surface boundary elements, where as Mesh B was based on 47 nodes and 29 surface boundary elements. Mesh $\mathrm{C}$, used for p-refinement approach, was defined with 28 nodes and 16 surface boundary elements.

Figure 7 shows the logarithmic evolution of the global estimator in expression (18), versus the logarithm of $1 / \mathrm{NDF}$.

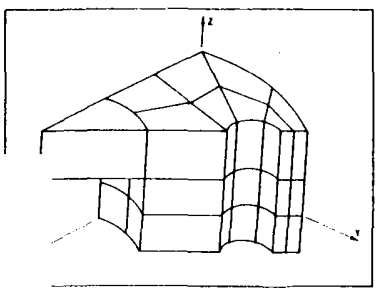

(a)

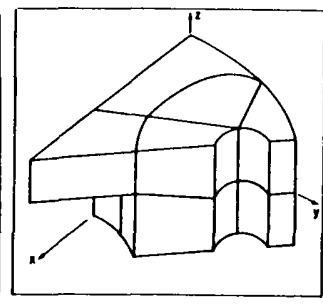

(b)

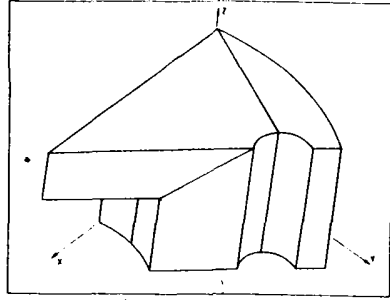

(c)

Fig. 6. Pressure vessel discretisations:

(a) Mesh A

(b) Mesh B

(c) Mesh C

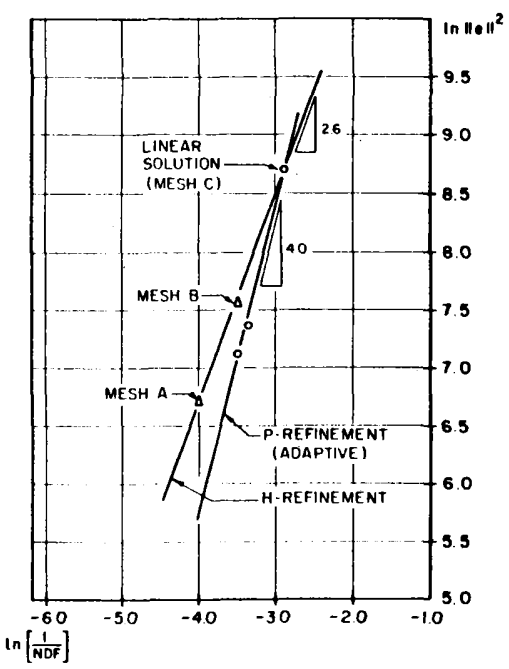

Fig. 7. Global estimator convergence comparison for pressure vessel analysis. 
The p-adaptive approach for the BEM has been presented and it seems to be a promising area in numerical methods for the analysis of complex engineering problems.

The p-adaptive convergence rates have showed better perfor mance than the $\mathrm{h}$-convergence ones in all the cases analysed herein, although the convergence criteria (in terms of indicators and estimators) are still under study and more mathematical research is needed for their consolidation.

The method proposed here has demostrated versatility and his advantages are twofold: the human effort to prepare input data are greatly reduced and, since the refinement in selective (either automatic or interactive), the user is able to master the degree of aproximation required, eiter in terms of predefined cost or computer time.

\section{ACKNOWLEDGMENTS}

This work was possible thanks to grant $\mathrm{N}$ ㅇ 1306-82 of the Com. Asesora de Investigación Cientifica y Técnica (CAICYT) of the Spanish Ministry of Education and Research (MEC).

REFERENCES

1. Rank, E.: "A-posteriori-Fehlerabschätzungen und adaptive Netzverfeinerung für Finite-Element-und Randintegralelement-Methoden", PH. D. Thesis, Technische Univ. München, 1985.

2. Alarcón, E., Abia, L, and Reverter, A.: "On the possibility of adaptive boundary elements", in Accuracy Estimates and Adaptive Refinements in Finite Elements Computations (ARFEC), Lisbon, 1984 .

3. Alarcón, E., Reverter, A., and Molina, J.: "Hierarchical Boundary Elements", Comp. Struc., 20(1-3) 151-156. (1985).

4. Alarcón, E. and Reverter, A.: "p-adaptive Boundary Elements", Int. J. Num. Meth. Eng.. To be published.

5. Cerrolaza, M. and Alarcón, E.: "p-adaptive Boundary Elements in 3-D potential problems", to be published in Comm. in Applied Numerical Methods.

6. Peano, A.G.: "Hierarchies of conforming finite elements", Doctoral Dissert., Washington Univ., St. Louis (1975). 
7. Babuska, K. and Rheinboldt, W.C.: "A posteriori error estimates for the FEM", Int. J. Num. Meth. Eng., 12, 1557-1616. (1978).

8. Kelly, D.W., Gago, J., Zienkiewicz, O.C. and Babuska, I.: "A posteriori error analysis and adaptive processes in the FEM", parts. I\&II, Int. J. Num. Meth. Eng., 19, 1593-1619. (1983).

9. Szabo, B.A., Basu, P.K., and Rosow, M.P.: "Adaptive finite element based on p-convergence", NASA Conf. Pub. 2059, pp. 43-50. (1978).

10. Arnold, D.N. and Wendland, W.L.: "On the asymptotic convergence of collocation methods", Preprint 665, TH Darmstad, Dept. of Mathematics, Fed. Rep. Germany. (1982).

11. Babuska, I.: "The selfadaptive approach in the FEM", in Mathematics of Finite Elements and Applications, (J.R. Whi teman, Ed.), New York, Academic Press. (1975). 